\section{(6) OPEN ACCESS}

\title{
The prospective association between childhood cognitive ability and somatic symptoms and syndromes in adulthood: the 1958 British birth cohort
}

\author{
Eva M Kingma, ${ }^{1}$ Judith G M Rosmalen, ${ }^{1}$ Peter D White, ${ }^{2}$ Stephen A Stansfeld, ${ }^{2}$ \\ Charlotte Clark ${ }^{2}$
}

\begin{abstract}
${ }^{1}$ University of Groningen, University Medical Center Groningen, Department of Psychiatry, Interdisciplinary Center Psychopathology and Emotion regulation (ICPE), Groningen, The Netherlands ${ }^{2}$ Centre for Psychiatry, Barts \& The London School of Medicine \& Dentistry, Queen Mary University of London, London, UK
\end{abstract}

\section{Correspondence to}

Dr Charlotte Clark, Centre for Psychiatry, Barts \& The London School of Medicine \& Dentistry, Queen Mary University of London, First Floor, Old Anatomy Building, Charterhouse Square, London EC1M 6BQ, UK; c.clark@qmul.ac.uk

Received 9 May 2013 Accepted 18 July 2013 Published Online First 10 September 2013

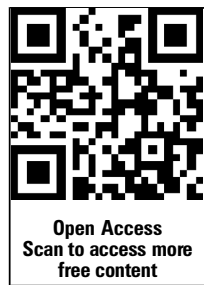

To cite: Kingma EM, Rosmalen JGM, White PD, et al. J Epidemiol Community Health 2013;67:1047-1053.

\begin{abstract}
Background Cognitive ability is negatively associated with functional somatic symptoms (FSS) in childhood. Lower childhood cognitive ability might also predict FSS and functional somatic syndromes in adulthood. However, it is unknown whether this association would be modified by subjective and objective measures of parental academic expectations.
\end{abstract}

Methods 14068 participants from the 1958 British birth cohort, whose cognitive ability was assessed at 11 years. Outcomes were somatic symptoms at 23, 33 and 42 years. Self-reported irritable bowel syndrome (IBS), chronic fatigue syndrome/myalgic encephalomyelitis (CFS/ME) and operationally defined CFS-like illness were measured at 42 years.

Results Lower cognitive ability at age 11 years was associated with somatic symptoms at ages 23,33 and 42 years. Adjusting for sex, childhood internalising problems, previous somatic symptoms and concurrent psychological symptoms, childhood cognitive ability remained negatively associated with somatic symptoms at age 23 years $(\beta=-0.060,95 \% \mathrm{Cl}-0.081$ to $-0.039, p<0.01), 33$ years $(\beta=-0.031,95 \% \mathrm{Cl}$ -0.050 to $-0.011, p<0.01$ ), but not with somatic symptoms at 42 years. Overall, we found no clear association between lower childhood cognitive ability and CFS/ME, CFS-like illness and IBS. Associations between cognitive ability and somatic symptoms at 23 years were moderated by low parental social class, but not by subjective indicators of parental academic expectations.

Conclusions Lower childhood cognitive ability predicted somatic symptoms, but not CFS/ME, CFS-like illness and IBS in adulthood. While earlier research indicated an important role for high parental academic expectations in the development of early-life FSS, these expectations do not seem relevant for somatic symptoms or functional somatic syndromes in later adulthood.

\section{INTRODUCTION}

Functional somatic symptoms (FSS), often also referred to as medically unexplained symptoms, are symptoms that cannot be fully explained by underlying organic pathology or conventionally defined medical disease. ${ }^{1}$ FSS are common both in childhood $^{2} 3$ and adulthood, ${ }^{45}$ and can be functionally impairing. ${ }^{6}$ The aetiology of FSS is poorly understood, but FSS are known to result from multifactorial processes, including complex interactions between biological, psychological and social vulnerability factors. ${ }^{8-10}$ One vulnerability factor for FSS might be cognitive ability. Previous studies have shown that cognitive ability is negatively associated with FSS in adults. ${ }^{11-13}$ However, these studies cannot exclude reverse causality in which FSS negatively influence performance on cognitive ability tests. One study related lower childhood cognitive ability to the development of FSS during adolescence, ${ }^{14}$ suggesting that cognitive ability is a risk factor for FSS early in life. It is plausible that the effect of childhood cognitive ability on FSS extends into adulthood. This is supported by two studies to date: adult men with lower cognitive ability on conscription into military service were more likely to report having globus sensation ${ }^{13}$ and lower cognitive ability scores in childhood were associated with chronic widespread pain at the age of $45 .{ }^{15}$

The mechanisms responsible for the association between lower cognitive ability and FSS remain unclear. Candidate mechanisms in adults might include psychological distress, which has been associated with FSS and with clusters of related FSS; that is, functional somatic syndromes or disorders (FSD) in adulthood. ${ }^{16-18}$ However, findings from studies of the aetiology of FSS in adults cannot be generalised to children and vice versa; there may be specific explanatory factors across the lifespan. Previous studies have indicated an important influence for parental factors in the relationship between childhood cognitive ability and childhood FSS and FSD. Lower cognitive ability has been significantly associated with FSS during adolescence for adolescents perceiving high parental academic expectations of their achievements, but not for those perceiving low parental academic expectations. ${ }^{14}$ In chronic fatigue syndrome (CFS), a type of FSD, it was shown that parental expectations of the cognitive ability of children with CFS were higher than parental expectations of the cognitive ability of children without CFS. ${ }^{19}$ Parental expectations might influence the association between cognitive abilities and FSS, since lower cognitive ability in combination with high parental expectations might particularly result in distress. Circumstantial evidence for a moderating role for parental factors comes from studies on the association between child IQ and emotional problems. While lower IQ scores in children have been associated with an increased risk of emotional problems, ${ }^{20}$ children whose parents had a higher mean IQ had more 
emotional symptoms, even after adjusting for the child's own IQ. ${ }^{21} 22$ This suggests moderation by high parental IQ and perhaps more broadly by high parental social class. Whether these findings can be applied to FSS is unknown. While children with lower cognitive ability are at risk for FSS in childhood ${ }^{14}$ and potentially for FSS and FSD in adulthood, it is unknown whether mechanisms making these children vulnerable to earlylife FSS are still relevant in adulthood.

Our aim was to study the prospective association between childhood cognitive ability with FSS and FSD in adulthood. We hypothesised that lower childhood cognitive ability increases the risk for FSS and FSD in adulthood and that this association would be modified by parental factors, especially high parental academic expectations as subjectively perceived by the child, the parents and the teacher, and as objectively reflected in high parental social class.

\section{METHODS}

\section{Population}

Data were from the 1958 Birth Cohort study. This longitudinal study follows 17638 individuals born in England, Scotland and Wales in a single week in 1958. Childhood data were obtained from the mother, teacher and participant at 7,11 and 16 years. Adulthood data were gathered at 23, 33 and 42 years. At 7 years, $92.2 \%$ of the eligible sample participated; at 11 years $91.5 \%$; at 16 years $86.7 \%$; at 23 years $76.1 \%$; at 33 years $70.7 \%$ and at 42 years $70.8 \% .^{23}$

\section{Childhood cognitive ability}

At 11 years, childhood cognitive ability was measured using a general ability test devised by the National Foundation for Educational Research in England and Wales, ${ }^{24}$ which shows validity with scores used to select 11-year-olds for secondary school. $^{24}$ The general ability test is composed of 40 verbal and 40 non-verbal items. Children were presented with an example of four words/shapes/symbols that were linked logically, semantically or phonologically, and they were then given another set of three words, shapes or symbols with a blank space. Participants were required to select the missing word/shape/ symbol from a list of five alternatives.

\section{Somatic symptoms in adulthood}

Somatic symptoms in adulthood were assessed using the eight somatic items from the Malaise Inventory at ages 23, 33 and 42 years. $^{25-27}$ These included the questions: 'Do you often have back-ache?', 'Do you often have bad headaches?', 'Have you at times had a twitching of the face, head or shoulders?', 'Do you suffer from indigestion?', 'Do you suffer from an upset stomach?', 'Does your heart often race like mad?', 'Do you have bad pains in your eyes?' and 'Are you troubled with rheumatism/ fibrositis?' (yes/no). The measure does not determine whether these somatic symptoms are functional, but previous research has shown that higher somatic symptom scores are likely to represent FSS. ${ }^{28} 29$ The somatic symptoms form scales of low-to-moderate reliability in our analyses (23 years Cronbach's $\alpha=0.50, n=12398,33$ years Cronbach's $\alpha=0.54, n=11272$, 42 years Cronbach's $\alpha=0.57, \mathrm{n}=11276$ ) and in previous analyses. ${ }^{27}$ Removing any of the items did not result in higher internal consistencies; thus, the moderate reliability is inherent to the influence of the individual symptoms.

\section{FSD in adulthood}

At 42 years, self-reported diagnoses of irritable bowel syndrome (IBS) and CFS or myalgic encephalomyelitis (CFS/ME) were available: 'Have you ever had irritable bowel syndrome?', 'Have you ever had chronic fatigue syndrome (CFS)/myalgic encephalomyelitis (ME)?' CFS-like illness was defined independently of self-reported diagnosis at 42 years. The criteria used were broadly equivalent to the Centers for Disease Control and Prevention criteria for CFS, ${ }^{30}$ including a self-report of 'feeling tired most of the time', in addition to $\geq 4$ from the following symptoms from the Malaise Inventory ${ }^{25}$ : 'bad headaches', 'difficulty falling asleep/staying asleep', 'backache', 'rheumatism/ fibrositis' and 'being unable to concentrate' from the 12-item General Health Questionnaire. ${ }^{31}$ All the three measures have been used in previous research. ${ }^{32}$

\section{Potential moderators}

Parental academic expectations as perceived by the child: at age 16, the participants were asked 'How anxious do you think your parents are that you should do well at school?': (1) very anxious, (2) fairly anxious, (3) contented if I do my best, (4) they do not mind one way or the other and (5) uncertain. Educational aspirations: at age 16, parents were asked 'which of the following would they like the study child to do': (1) leave at minimum school-leaving age (ie, end of this school year); (2) stay in full-time education beyond minimum school-leaving age, but not beyond 18 ; 3 ) continue some form of full-time education beyond age of 18 and (4) uncertain. Parental interest in child's education: at 16 years, teachers were asked 'with regard to the child's education, does the parent appear': (1) over concerned about the child's progress/expecting too high a standard; (2) very interested; (3) to show some interest; (4) to show little or no interest; (5) cannot say and (6) inapplicable (eg, no father/ father figure or mother/mother figure). Parental social class: parental social class was based on the father's social class, measured at 11 years, using the British Registrar General classification ${ }^{33}$ : I and II professional/managerial/technical, III other non-manual, III skilled manual and IV and V unskilled manual).

\section{Internalising problems in childhood}

We consider potential confounding of the association between childhood cognitive ability and adulthood somatic symptoms and FSD by internalising problems in childhood, which are associated with childhood cognitive ability ${ }^{34}$ and FSS. ${ }^{18} 35$ Internalising problems at age 11 years were measured using the teacher-rated Bristol Social Adjustment Guides. ${ }^{36}$ A continuous scale was formed by summing and square-root transforming the syndrome scores for unforthcomingness, withdrawal, depression and miscellaneous symptoms for internalising problems. ${ }^{37}$ A reliable scale was derived for internalising problems at age 11 years (Cronbach's $\alpha=0.69, \mathrm{n}=8046$ ).

\section{Psychological symptoms in adulthood}

To investigate whether the association between cognitive ability and somatic symptoms and FSD is influenced by comorbid psychological symptoms, we included scores from the 15 psychological items from the Malaise Inventory at ages 23, 33 and 42 years. $^{25}$ These include items such as 'Do you often get worried about things?' and 'Do you often feel miserable or depressed?'

\section{Statistical analyses}

Analyses were performed using the Statistical Package for the Social Sciences V.18.0 (SPSS Inc, Chicago, Illinois, USA). Descriptive statistics examined sex differences in cognitive ability, somatic symptoms and FSD (CFS/ME, CFS-like illness, IBS). Symptom sum scores were log transformed to normally 
Table 1 Sample characteristics

\begin{tabular}{|c|c|c|c|c|}
\hline & \multicolumn{4}{|l|}{ All $(\mathrm{N}=14$ 068) } \\
\hline & & Males $(51.3 \%)$ & Females $(48.7 \%)$ & Test statistics $†$ \\
\hline Mean cognitive ability (SD)‡ & $43.14(15.91)$ & $42.01(16.07)$ & $44.34(15.66)$ & $\mathrm{t}=-8.71^{* *}$ \\
\hline Mean verbal sub score (SD) $\ddagger$ & $22.16(9.26)$ & $21.15(9.37)$ & $23.23(9.02)$ & $\mathrm{t}=-13.44^{* *}$ \\
\hline Mean non-verbal sub score (SD)‡ & $20.98(7.49)$ & $20.86(7.56)$ & $21.11(7.43)$ & $t=-1.94^{*}$ \\
\hline \multicolumn{5}{|l|}{ Internalising problemsł } \\
\hline Mean score (SD) & $3.58(4.24)$ & $3.83(4.28)$ & $3.31(4.18)$ & $z=-10.20^{* *}$ \\
\hline Somatic symptom $\neq$ score mean (SD) & $0.32(0.55)$ & $0.29(0.54)$ & $0.34(0.57)$ & $z=-4.49^{* *}$ \\
\hline Gastric symptom‡ score mean (SD) & $0.25(0.52)$ & $0.20(0.46)$ & $0.31(0.57)$ & $z=-12.36^{* *}$ \\
\hline \multicolumn{5}{|l|}{ Somatic symptom scores in adulthood } \\
\hline Mean score (SD) at age 23 years & $0.76(1.09)$ & $0.63(1.00)$ & $0.90(1.16)$ & $z=-14.23^{* *}$ \\
\hline Mean score (SD) at age 33 years & $0.81(1.14)$ & $0.74(1.10)$ & $0.88(1.17)$ & $z=-7.19^{* *}$ \\
\hline Mean score (SD) at age 42 years & $1.01(1.28)$ & $0.92(1.23)$ & $1.11(1.31)$ & $\mathrm{z}=-8.23^{* *}$ \\
\hline \multicolumn{5}{|l|}{ Psychological symptom scores } \\
\hline Mean score (SD) at age 23 years & $1.92(2.23)$ & $1.37(1.83)$ & $2.46(2.46)$ & $z=-26.03^{* *}$ \\
\hline Mean score (SD) at age 33 years & $1.57(2.18)$ & $1.25(1.89)$ & $1.89(2.38)$ & $z=-15.17^{* *}$ \\
\hline Mean score (SD) at age 42 years & $2.47(2.63)$ & $2.11(2.45)$ & $2.81(2.75)$ & $z=-14.24^{* *}$ \\
\hline CFS at age 42 years & & & & $\chi^{2}=3.83^{*}$ \\
\hline Yes $(\mathrm{N}, \%)$ & $103(0.7)$ & $41(0.6)$ & $62(0.9)$ & \\
\hline No $(\mathrm{N}, \%)$ & $9670(68.7)$ & $4786(66.3)$ & $4946(72.2)$ & \\
\hline CFS-like illness at age 42 years & & & & $\chi^{2}=34.65^{* *}$ \\
\hline Yes $(\mathrm{N}, \%)$ & $196(1.4)$ & $56(0.8)$ & $140(2.0)$ & \\
\hline No $(N, \%)$ & $9593(68.2)$ & $4834(66.9)$ & $4955(72.4)$ & \\
\hline IBS at age 42 years & & & & $\chi^{2}=171.23^{* *}$ \\
\hline Yes $(\mathrm{N}, \%)$ & $830(5.9)$ & $230(3.2)$ & $600(8.8)$ & \\
\hline No $(N, \%)$ & $8352(59.4)$ & $4533(62.8)$ & $4649(67.9)$ & \\
\hline Parental academic expectations§ (\%) & & & & $\chi^{2}=214.28^{* *}$ \\
\hline Very anxious & 17.5 & 20.8 & 14.0 & \\
\hline Fairly anxious & 11.3 & 12.6 & 10.0 & \\
\hline Content if tries & 39.4 & 34.3 & 44.8 & \\
\hline Not bothered & 3.5 & 3.3 & 3.7 & \\
\hline Uncertain & 2.1 & 2.3 & 1.8 & \\
\hline Educational aspirations of parents§ (\%) & & & & $\chi^{2}=48.56^{* *}$ \\
\hline Leave at a minimum age & 25.7 & 27.4 & 24.0 & \\
\hline Continue full-time education to 18 years & 18.1 & 16.2 & 20.2 & \\
\hline Continue full-time education after 18 years & 23.4 & 23.5 & 23.3 & \\
\hline Uncertain & 4.3 & 4.4 & 4.3 & \\
\hline \multicolumn{5}{|l|}{ Parental interest in child's education§ } \\
\hline Mother (\%) & & & & $\chi^{2}=28.51 * *$ \\
\hline Over concerned & 1.4 & 1.7 & 1.1 & \\
\hline Very interested & 25.8 & 24.7 & 27.1 & \\
\hline Some interest & 22.6 & 21.9 & 23.4 & \\
\hline Little or no interest & 11.8 & 12.5 & 11.1 & \\
\hline Cannot say & 9.0 & 9.4 & 8.6 & \\
\hline Inapplicable & 1.0 & 1.1 & .9 & \\
\hline Father (\%) & & & & $28.36^{* *}$ \\
\hline Over concerned & 1.5 & 1.9 & 1.1 & \\
\hline Very interested & 22.2 & 22.1 & 22.3 & \\
\hline Some interest & 19.1 & 19.1 & 19.1 & \\
\hline Little or no interest & 12.3 & 13.2 & 11.4 & \\
\hline Cannot say & 11.0 & 10.4 & 11.7 & \\
\hline Inapplicable & 2.5 & 2.5 & 2.5 & \\
\hline Parental social class $¥$ (\%) & & & & $\chi^{2}=.80$ \\
\hline I/II professional/managerial/technical & 20.9 & 20.6 & 21.3 & \\
\hline III other non-manual & 8.2 & 8.1 & 8.3 & \\
\hline III skilled manual & 37.9 & 38.0 & 37.8 & \\
\hline $\mathrm{V}$ and $\mathrm{V}$ unskilled manual & 20.6 & 20.5 & 20.7 & \\
\hline
\end{tabular}


distributed variables when used as dependent variables in the analyses. Sex-adjusted linear and logistic regression analyses examined the associations between childhood cognitive ability and somatic symptoms at ages 23, 33 and 42 years, CFS/ME, CFS-like illness and IBS at 42 years. Analyses were further adjusted for internalising problems at age 11 years and somatic symptoms assessed at the sweep prior to the outcome. For somatic symptoms at age 23 years, somatic and gastrointestinal symptoms assessed at 16 years were included. Analyses were additionally adjusted for psychological symptoms at the same sweep at which the outcome variable was measured. In addition, when there was a significant interaction between the potential moderators and childhood cognitive ability in predicting somatic symptoms, further analyses were stratified based on that moderator. The heterogeneity between the adjusted regression coefficients for the association between cognitive ability and somatic symptoms at 23,33 and 42 years was investigated using the metan command in STATA V.12 (Stata Corp, College Station, Texas, USA). Complete case analyses are presented, which showed similar associations as analyses carried out using multiple imputation in STATA (V.12). Analyses are therefore presented for participants who had complete data on childhood cognitive ability at age 11 years $(\mathrm{N}=14068 ; 91.7 \%$ of all participants at age 11 years).

\section{RESULTS}

\section{Study population}

The characteristics of our study population can be found in table 1 . The mean symptom scores increase slightly over time. Most scores were statistically significantly higher for women, including cognitive ability scores, somatic symptoms, psychological symptoms and prevalence of FSD. However, men had significantly higher scores on internalising problems, and more often perceived higher parental expectations and parental overconcern.

\section{Associations between childhood cognitive ability and somatic symptoms and FSD in adulthood}

Table 2 shows multivariable associations between childhood cognitive ability at age 11 years and somatic symptoms at ages 23, 33 and 42 years, adjusted for sex. Childhood cognitive ability was significantly and negatively associated with somatic symptom scores. These associations decreased slightly over time. After further adjusting for internalising problems at age 11 years and previous somatic symptoms, the effects of childhood cognitive ability on somatic symptoms at ages 23, 33 and 42 years attenuated, but remained significant. Additionally, adjusting for psychological symptoms attenuated the association between cognitive ability and somatic symptoms at 23 years $(\beta=-0.060$, $95 \%$ CI -0.081 to $-0.039, \quad \mathrm{p}<0.01)$ and at 33 years $(\beta=-0.031,95 \% \mathrm{CI}-0.050$ to $-0.011, \mathrm{p}<0.01)$, but led to a non-significant association at 42 years $(\beta=-0.016$, 95\% CI -0.034 to $0.002, p=0.08)$. There was significant heterogeneity between the adjusted regression coefficients for the effect of cognitive ability on somatic symptoms at 23,33 and 42 years (heterogeneity $\chi^{2}=9.83, \mathrm{df}=2, \mathrm{p}=0.0007$ ), reflecting that the effect of cognitive ability on somatic symptoms did differ over adulthood.

Table 2 also shows multivariable associations between childhood cognitive ability at age 11 years and CFS/ME, CFS-like illness and IBS, adjusted for sex. Childhood cognitive ability was significantly and negatively associated with CFS-like illness, but not with self-reported CFS/ME and IBS. After further adjusting for internalising problems at age 11 years and previous

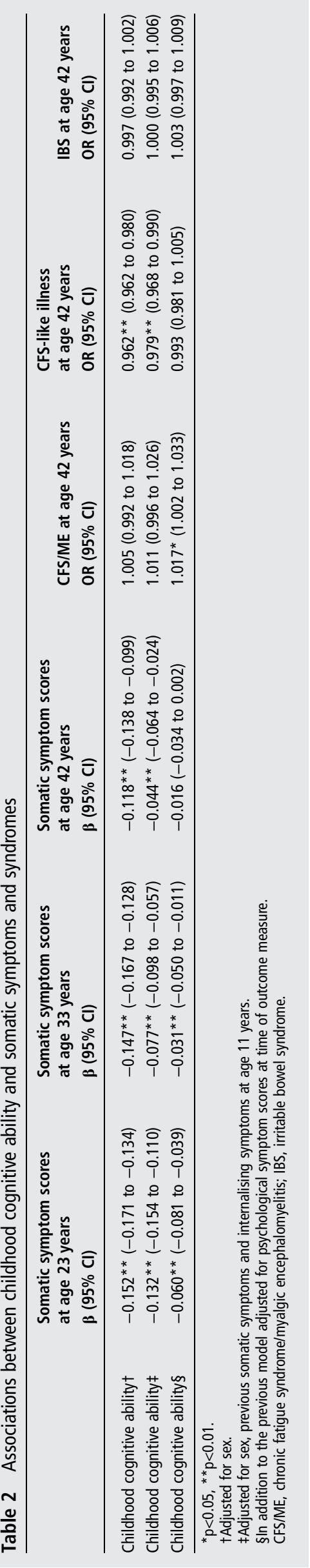


somatic symptoms, the effect of childhood cognitive ability on CFS-like illness at age 42 years attenuated, but remained significant. Including psychological symptoms in the model led to a non-significant association between childhood cognitive ability and CFS-like illness $(\mathrm{OR}=0.993,95 \%$ CI 0.981 to 1.005 $\mathrm{p}=0.237$ ). It resulted in a small but significant positive association between cognitive ability and self-reported CFS/ME $(\mathrm{OR}=1.017,95 \% \mathrm{CI} 1.002$ to $1.033, \mathrm{p}<0.05)$, but a nonsignificant association between cognitive ability and IBS $(\mathrm{OR}=1.003,95 \% \mathrm{CI} 0.997$ to $1.009, \mathrm{p}=0.289)$.

\section{Parental modifying factors}

No significant interactions were found between childhood cognitive ability and the three subjective indicators of parental academic expectations (parental academic expectations as perceived by the child, parental interest reported by the teacher and parental aspirations of the child's future academic performance) in predicting somatic symptoms at age 23,33 or 42 years. A significant interaction was found between childhood cognitive ability and parental social class in predicting somatic symptoms at age 23 years. Table 3 shows an overall increase in the inverse association between cognitive ability and somatic symptoms at age 23 years (adjusted for sex, previous somatic symptoms, internalising problems at age 11 and psychological symptoms at age 23 years) with decreasing parental social class. In contrast, no significant interaction was found between childhood cognitive ability and parental social class in predicting somatic symptoms at age 33 or 42 years.

\section{DISCUSSION}

In this prospective general population study, lower cognitive ability at age 11 years was associated with somatic symptoms at ages 23,33 and 42 years. These associations, with the exception of somatic symptoms at 42 years, were independent of sex, somatic symptoms at the previous sweep, internalising problems in childhood and concurrent psychological symptoms.

Lower cognitive ability at 11 years was not associated with selfreported IBS but was associated with CFS-like illness at 42 years: however, this association became non-significant after full adjustment. We found a small significant association between higher childhood cognitive ability and self-reported CFS/ME at 42 years, but only after full adjustment for sex, somatic symptoms at the previous sweep, internalising problems in childhood and concurrent psychological symptoms. Associations between childhood cognitive ability and somatic symptoms in adulthood were not moderated by high parental academic expectations as subjectively perceived by the child, the parents or the teacher.

Table 3 Stratified multivariable associations between childhood cognitive ability and somatic symptoms

\begin{tabular}{|c|c|}
\hline Group & $\begin{array}{l}\text { Somatic symptom scores } \\
\text { at age } 23 \text { years } \\
\beta(95 \% \mathrm{Cl})\end{array}$ \\
\hline All childhood cognitive ability & $-0.060^{* *}(-0.081$ to -0.039$)$ \\
\hline \multicolumn{2}{|l|}{ Parental social class at age 11 years } \\
\hline I/II professional/managerial/technical & $-0.041(-0.083$ to 0.002$)$ \\
\hline III non-manual & $-0.037(-0.014$ to 0.030$)$ \\
\hline III skilled manual & $-0.048^{* *}(-0.080$ to -0.015$)$ \\
\hline IV/V unskilled manual & $-0.068^{* *}(-0.113$ to -0.023$)$ \\
\hline
\end{tabular}

The association between childhood cognitive ability and somatic symptoms at 23 years increased with decreasing parental social class.

The principal strengths of this study lie in its large population-based sample and its prospective design, including somatic symptoms measured at several time-points. With regard to parental academic expectations, data were used from multiple informants, namely children, parents and teachers. Additionally, parental social class was used as an objective indicator for parental academic expectations. There were several limitations. It was not clear whether all somatic symptoms were functional. The internal consistencies of the somatic item scale of the Malaise Inventory were low to moderate, which means that the associations observed may be influenced by the unreliable measurement of somatic symptoms. However, the internal consistencies are close to the figures reported previously for the Malaise Inventory. ${ }^{26}$ When studying large cohorts, there is an inevitable risk of missing data, but repeating main analyses after multiple imputations indicated similar findings. Assessment of CFS/ME and IBS was primarily by self-report and not derived using clinical diagnoses or severity criteria for CFS/ME or IBS.

This study investigated the prospective association between childhood cognitive ability and several somatic symptoms and FSD in adulthood, while adjusting for several potential life course confounders. Lower childhood cognitive ability was associated with somatic symptoms at 23 and 33 years, but not with somatic symptoms at 42 years, after full adjustment. The significant associations between cognitive ability and somatic symptoms are in line with previous studies on cognitive ability and FSS in adults. ${ }^{11-13}$ Although findings in adults cannot exclude reverse causality, our findings suggest that lower cognitive ability precedes the development of FSS many years later. This is supported by previous findings that found an association between lower cognitive ability in early life and FSS in adulthood. ${ }^{13} 15$ Psychological symptoms confounded part of the association between childhood cognitive ability and somatic symptoms at 23 and 33 years, and led to non-significant associations between childhood cognitive ability and somatic symptoms and CFS-like illness at 42 years. Psychological distress in adulthood, associated with childhood cognitive ability in previous studies, ${ }^{38}$ may mediate the association between lower childhood cognitive ability and somatic symptoms. Despite this, we still found that lower childhood cognitive ability remained an independent predictor for somatic symptoms in early adulthood. Overall, no clear association was found between cognitive ability and selfreported FSD in the fully adjusted models. We found an association between higher cognitive ability and self-reported CFS/ $\mathrm{ME}$, which was in contrast to our hypothesis. Since the association was only found when additionally adjusting for psychological symptoms and since it was rather small, we might consider this as a chance finding. Compared with CFS/ME, no positive association was found between childhood cognitive ability and CFS-like illness. A difference in the association between childhood cognitive ability and CFS/ME versus CFS-like illness may be partly explained by the fact that we used different definitions of CFS-that is self-reported versus operationalised diagnosis. ${ }^{31}$

Parental academic expectations as perceived by the child, the teacher and the parent did not moderate the association between childhood cognitive ability and somatic symptoms in adulthood. Although earlier analysis of the TRAILS adolescent cohort showed an important role for high parental expectations in relation to cognitive ability and FSS in childhood, ${ }^{14}$ the current study suggests that this effect does not extend into 
adulthood. However, whereas the TRAILS cohort assessed parental academic expectations as perceived by the child, the 1958 birth cohort data included information on educational expectations derived from multiple informants including the parent, the teacher and the child. Therefore, the measures in both studies may not be directly comparable. It is, however, possible that parental factors may only directly affect the development of FSS in children and this should be the focus of further research. The question arises as to which mechanisms explain the long-lasting association of lower childhood cognitive ability with somatic symptoms in adulthood. The association may be spurious in that lower cognitive ability in childhood may be confounded by a related but unmeasured variable. If it is aetiologically important, it may be that children with higher cognitive ability develop better coping strategies to deal with stressful situations that prevent them from developing FSS. Previous studies in children showed more internal coping strategies in children with higher cognitive ability. ${ }^{39}$ As mentioned, lower childhood cognitive ability was associated with psychological distress in early adulthood, ${ }^{38}$ increasing vulnerability to FSS. Whereas high parental expectations might be a source of distress that is important in relation to FSS in early life, other more proximal sources of distress might be relevant in making persons with lower cognitive ability vulnerable to FSS in adulthood. Such a proximal source of distress may be unemployment: being unwillingly unemployed explained part of the association between cognitive ability and FSS in adults. ${ }^{11}$ The association between cognitive ability and somatic symptoms increased with decreasing parental social class, but only at age 23 years. Children with lower parental social class may be more likely to have a similar lower social class in adulthood, increasing the risk of experienced symptoms in adulthood. ${ }^{40}$

In conclusion, lower childhood cognitive ability may be a vulnerability factor for FSS at least until early adulthood, but not for self-reported FSD in later life. The mechanisms that might explain the association between childhood cognitive ability and FSS should be studied further.

\section{What is already known on this subject?}

- Functional somatic symptoms (FSS) are common both in childhood and adulthood, yet their aetiology is poorly understood.

- Studies have found an association between lower cognitive ability and FSS in childhood; indicating an important influence for parental factors in this association.

- It is plausible that the effect of childhood cognitive ability on FSS extends into adulthood, but it is unknown whether mechanisms making children vulnerable to early-life FSS are still relevant in adulthood.

Acknowledgements The authors would like to thank Professor Joan Morris (Queen Mary University of London) for statistical advice and for conducting the meta-analysis presented in the paper and Jon Johnson (Institute of Education) for data assistance.

Contributors EMK, CC, PDW, SAS and JGMR have contributed to the conception and design of this paper. Analyses were undertaken by EMK and supervised by CC. EMK, CC, PDW, SAS and JGMR have contributed to the interpretation of the findings, drafts and revisions of the paper. EMK, CC, PDW, SAS and JGMR have approved the final version of this manuscript.

\section{What this study adds?}

- This study supports the findings that lower cognitive ability in childhood precedes the development of FSS many years later in adulthood.

- While earlier research indicated an important role for high parental academic expectations in the development of early-life FSS, these expectations do not seem relevant for somatic symptoms or functional somatic syndromes in later adulthood.

- Psychological symptoms also play a role in the association between childhood cognitive ability and adulthood somatic symptoms.

- Further studies of factors that might explain the association between childhood cognitive ability and FSS in adulthood, such as coping strategies and psychological distress, should be conducted to inform preventive interventions for FSS.

Competing interests PDW has carried out consultancy work for the UK government and a reinsurance company.

Ethics approval North Thames Multi-Centre Research Ethics Committee.

Provenance and peer review Not commissioned; externally peer reviewed.

Data sharing statement There are no additional unpublished data from the study_all data are already available on the UK Data Archive.

Open Access This is an Open Access article distributed in accordance with the Creative Commons Attribution Non Commercial (CC BY-NC 3.0) license, which permits others to distribute, remix, adapt, build upon this work non-commercially, and license their derivative works on different terms, provided the original work is properly cited and the use is non-commercial. See: http://creativecommons.org/ licenses/by-nc/3.0/

\section{REFERENCES}

1 Wessely S, Nimnuan C, Sharpe M. Functional somatic syndromes: one or many? Lancet 1999;354:936-9.

2 Janssens KAM, Rosmalen JGM, Ormel J, et al. Pubertal status predicts back pain, overtiredness, and dizziness in American and Dutch adolescents. Pediatrics 2011;128:553-9.

3 Perquin CW, Hazebroek-Kampschreur AAJM, Hunfeld JAM, et al. Pain in children and adolescents: a common experience. Pain 2000;87:51-8.

4 Fink $P$, Rosendal M, Olesen $F$. Classification of somatization and functional somatic symptoms in primary care. Aust N Z J Psychiatry 2005;39:772-81.

5 Rosmalen JGM, Tak LM, de Jonge P. Empirical foundations for the diagnosis of somatization: implications for DSM-5. Psychol Med 2011;41:1133-42.

6 Barsky AJ, Borus JF. Somatization and medicalization in the era of managed care. JAMA 1995;274:1931-4

7 Smith GR, Monson RA, Ray DC. Patients with multiple unexplained symptomstheir characteristics, functional health, and health-care utilization. Arch Intern Med 1986; 146:69-72

8 Brown RJ. Psychological mechanisms of medically unexplained symptoms: an integrative conceptual model. Psychol Bull 2004;130:793-812.

9 Rief W, Broadbent E. Explaining medically unexplained symptoms-models and mechanisms. Clin Psychol Rev 2007;27:821-41.

10 Deary V, Chalder T, Sharpe M. The cognitive behavioural model of medically unexplained symptoms: a theoretical and empirical review. Clin Psychol Rev 2007:27:781-97.

11 Kingma EM, Tak LM, Huisman $M$, et al. Intelligence is negatively associated with the number of functional somatic symptoms. J Epidemiol Community Health 2009;63:900-U54.

12 Gale CR, Batty GD, Tynelius $P$, et al. Intelligence in early adulthood and subsequent hospitalization for mental disorders. Epidemiology 2010;21:70-7.

13 Gale CR, Wilson JA, Deary IJ. Globus sensation and psychopathology in men: the Vietnam experience study. Psychosom Med 2009;71:1026-31.

14 Kingma EM, Janssens KAM, Venema $M$, et al. Adolescents with low intelligence are at risk of functional somatic symptoms: the TRAILS study. J Adolesc Health 2011;49:621-6

15 Gale CR, Deary IJ, Cooper C, et al. Intelligence in childhood and chronic widespread pain in middle age: the National Child Development Survey. Pain 2012:153:2339-44 
16 Löwe B, Spitzer RL, Williams JBW, et al. Depression, anxiety and somatization in primary care: syndrome overlap and functional impairment. Gen Hosp Psychiatry 2008;30:191-9.

17 Henningsen P, Zimmermann T, Sattel H. Medically unexplained physical symptoms, anxiety, and depression: a meta-analytic review. Psychosom Med 2003:65:528-33.

18 Haug TT, Mykletun A, Dahl AA. The association between anxiety, depression, and somatic symptoms in a large population: the HUNT-II study. Psychosom Med 2004:66:845-51.

19 Godfrey E, Cleare A, Coddington A, et al. Chronic fatigue syndrome in adolescents: do parental expectations of their child's intellectual ability match the child's ability? J Psychosom Res 2009;67:165-8.

20 Fergusson DM, Horwood LJ, Ridder EM. Show me the child at seven II: childhood intelligence and later outcomes in adolescence and young adulthood. J Child Psychol Psychiatry 2005:46:850-8.

21 Goodman R, Simonoff E, Stevenson J. The impact of child IQ, parent IQ and sibling IQ on child behavioural deviance scores. J Child Psychol Psychiatry 1995:36:409-25.

22 Whitley E, Gale CR, Deary IJ, et al. Association of maternal and paternal IQ with offspring conduct, emotional, and attention problem scores. Transgenerational evidence from the 1958 British Birth Cohort Study. Arch Gen Psychiatry 2011;68:1032-8.

23 Atherton K, Fuller E, Shepherd P, et al. Loss and representativeness in a biomedica survey at age 45 years: 1958 British Birth Cohort. J Epidemiol Community Health 2008;62:216-23.

24 Douglas J. The home and the school: a study of ability and attainment in the primary school. London, England: MacGibbon \& Kee, 1964.

25 Rutter ML. Psycho-social disorders in childhood, and their outcome in adult life. J R Coll Physicians Lond 1970;4:211-18.

26 Grant G, Nolan M, Ellis N. A reappraisal of the malaise inventory. Soc Psychiatry Psychiatr Epidemiol 1990;25:170-8.

27 Rodgers B, Pickles A, Power $C$, et al. Validity of the malaise inventory in general population samples. Soc Psychiatry Psychiatr Epidemiol 1999;34:333-41.

28 Kroenke K, Mangelsdorff AD. Common symptoms in ambulatory care-incidence, evaluation, therapy, and outcome. Am J Med 1989:86:262-6.
29 Korber S, Frieser D, Steinbrecher N, et al. Classification characteristics of the Patient Health Questionnaire-15 for screening somatoform disorders in a primary care setting. J Psychosom Res 2011;71:142-7.

30 Reeves WC, Lloyd A, Vernon SD, et al. Identification of ambiguities in the 1994 chronic fatigue syndrome research case definition and recommendations for resolution. BMC Health Serv Res 2003;3:25.

31 Goldberg DP, Gater R, Sartorius N, et al. The validity of two versions of the GHQ in the WHO study of mental illness in general health care. Psychol Med 1997;27:191-7

32 Clark C, Goodwin L, Stansfeld SA, et al. Premorbid risk markers for chronic fatigue syndrome in the 1958 British birth cohort. Br J Psychiatry 2011;199:323-9.

33 Stansfeld SA, Clark C, Rodgers B, et al. Repeated exposure to socioeconomic disadvantage and health selection as life course pathways to mid-life depressive and anxiety disorders. Soc Psychiatry Psychiatr Epidemiol 2011:46:549-58.

34 Jokela M, Batty GD, Deary IJ, et al. Low childhood IQ and early adult mortality: the role of explanatory factors in the 1958 British Birth Cohort. Pediatrics 2009;124: e380-8.

35 Beck JE. A developmental perspective on functional somatic symptoms. J Pediatr Psychol 2008;33:547-62.

36 Stott DH. The social adjustment of children. 3rd ed. London, England: University of London Press, 1969.

37 Ghodsian M. Measuring behaviour in the school and home. In: Fogelman K.ed. Growing up in Britain. London, England: The Macmillan Press Ltd, 1983:329-38.

38 Gale CR, Hatch SL, Batty GD, et al. Intelligence in childhood and risk of psychological distress in adulthood: the 1958 National Child Developmental Survey and the 1970 British Cohort Study. Intelligence 2009;37:592-9.

39 Gale CR, Batty GD, Deary IJ. Locus of control at age 10 years and health outcomes and behaviors at age 30 years: the 1970 British Cohort Study. Psychosom Med 2008;70:397-403.

40 Power C, Stansfeld SA, Matthews S, et al. Childhood and adulthood risk factors for socio-economic differentials in psychological distress: evidence from the 1958 British birth cohort. Soc Sci Med 2002;55:1989-2004. 